\title{
Lessons learnt from a pilot of assessment for learning
}

\author{
Authors: Siu Pan Cho, ${ }^{A}$ David Parry ${ }^{B}$ and Winnie Wade ${ }^{C}$
}

A 12-month pilot was carried out on assessments for learning and assessments of learning as part of workplace-based assessments in postgraduate medical education. This was carried out in three regions and core medical trainees and higher specialty medical trainees participated. Focus groups and questionnaires were utilised to investigate the trainees' and trainers' experiences and perceptions of assessments for learning. The study demonstrated that the trainees and trainers perceived the newly introduced assessments for learning - supervised learning events (SLEs) - as learning tools. However, SLEs were often undertaken with no previous organisation and with no direct observation, regardless of the underlying purposes and methods of the WPBAs. There was a lack of, or delayed or non-specific, feedback following SLEs, which would have impeded its educational value. Trainee and trainer disengagement was one of the contributing factors. These findings are valuable in informing and facilitating future successful implementation of assessments for learning.

KEYWORDS: Workplace-based assessment, assessment for learning, supervised learning events, feedback

\section{Introduction}

In 2011, the General Medical Council (GMC) recommended a series of changes to the current use of workplace-based assessments (WPBAs) in postgraduate medical training in their discussion paper, Learning and assessment in the clinical environment: the way forward. ${ }^{1}$ The paper recommended the introduction of separate formative and summative assessments as part of the revision of current WPBAs.

The distinction was made between assessments for learning (formative assessments) and assessments of learning (summative assessments). ${ }^{2}$ The purpose of an assessment for learning is to provide teachers and learners with information that would facilitate further development. ${ }^{2,3}$

The GMC recommended the introduction of supervised learning events (SLEs), which are trainee-led formative assessments that aim to promote and facilitate learning. ${ }^{1}$

Authors: ${ }^{A}$ education fellow, Royal College of Physicians, London, UK; ${ }^{B}$ deputy director of education, Royal College of Physicians, London, UK; ' Cdirector of education, Royal College of Physicians, London, UK.
Following the recommendations of the GMC, a Joint Royal Colleges of Physicians Training Board (JRCPTB) Working Group proposed a revised WPBA system to be piloted in 2012 and 2013. ${ }^{4}$ Trainees are encouraged to identify learner-directed learning goals with their trainers before any SLE. Both trainees and trainers should subsequently identify opportunities that would facilitate the acquisition of these learning goals and are suitable for SLEs. SLEs provide opportunities for trainees and trainers to interact. Furthermore, SLEs intend to promote deeper learning through effective feedback and self-reflection. Trainees and trainers should formulate action plans with further learning goals following SLEs. ${ }^{1}$

The pilot SLEs continued to use the assessment methods of mini-clinical evaluation exercise (mini-CEX), case-based discussion (CbD) and acute care assessment tool (ACAT). These methods were retained because they had previously been demonstrated to be feasible, reliable and valid. ${ }^{5-10}$ The intention of SLEs is to enhance learning through self-reflection and effective feedback. ${ }^{11}$ The scoring system, which is part of the current WPBAs, was removed from the SLEs to promote self-reflection and feedback. The anchor statements on the SLEs have been retained to provide trainees with a clear indication of their level of development. SLEs will not contribute directly towards the decision process of the Annual Review of Competence Progression (ARCP).

Here, we focus on the evaluation of the use of SLEs in postgraduate medical education and explore lessons learnt from the pilot of these assessments for learning.

\section{Objectives of the study}

The pilot provided an opportunity for an evaluation of the application of assessments for learning in the clinical environment. The following aspects of SLEs were evaluated: feasibility; validity; educational impact; and the role of SLEs in the ARCP.

Educational impact requires a longitudinal study. Therefore, this study only reported the perception of trainees and trainers on the educational value of SLEs. Furthermore, the study explored whether SLEs were able to improve the trainees' and trainers' perceptions of WPBAs.

\section{Method}

The study was conducted over a 12-month period by the Education Department at the Royal College of Physicians (London). The pilot started in August 2012 and was carried out in Wales, East Midlands North and Northern regions, and 
Box 1. HST specialties that participated in the WPBA pilot

Acute internal medicine

Clinical genetics

Clinical neurophysiology

General internal medicine

Genitourinary medicine

Geriatric medicine

Infectious diseases

Neurology

Palliative medicine

HST = higher specialty training; WPBA = workplace-based assessments.

involved core medical training (CMT) and higher specialty training (HST) in nine medical specialties (Box 1).

Visits to each region were conducted in June and July 2012 before the start of the pilot to provide information for the trainees and the trainers on the pilot and evaluation. Additionally, written guidance on SLEs was provided to the trainees and the trainers.

All CMT trainees, HST trainees of the participating specialties and their trainers in the three regions were involved in the pilot. In total, 546 core medical trainees, 309 higher specialty trainees and 669 trainers participated.

A fully integrated mixed method study was devised to address the objectives. The study utilised focus groups and online questionnaires to collect data throughout the 12-month period (Table 1). All trainees and trainers in the three pilot regions were invited to complete the online questionnaires and to participate in the focus groups. Participation was voluntary and the participants were self-selected.

\section{Focus groups}

The focus groups and questionnaires were conducted with informed consent and the trainees and trainers were interviewed separately. Focus groups for trainees and trainers were formed from each of the three pilot regions during the early, middle and late stage of the pilot (Table 1). There were two additional final focus groups, which contained one group of trainees and one group of trainers and focused specifically on the ARCP process. The data from all of the focus groups were anonymised and transcribed for analysis.

\section{Questionnaires}

There were three questionnaires that were completed by participants during the 12-month period (Table 1). The questionnaires contained questions that provided Likert scale-based answers accompanied by free text boxes. This enabled quantitative and qualitative data to be collected that were anonymised. The first questionnaire focused on the trainees' and trainers' opinions of the pre-pilot WPBAs, whereas the second and third questionnaires focused on SLEs and the ARCP process, respectively. The trainers who participated in the third questionnaire were all ARCP panel members.

The data from the focus groups and the questionnaires yielded both quantitative and qualitative data. The qualitative data were analysed by coding for thematic analysis using the principles described by Cohen et al. ${ }^{12}$

\section{Results}

In total, 20 focus groups were conducted from November 2012 to August 2013 across the three pilot sites (Table 1). There was a total of 76 CMT and HST trainees in all of the focus groups during the pilot, whereas there was a total of 43 trainers in all of the focus groups during the pilot. These focus groups concentrated on the trainees' and trainers' experiences with SLEs. The ARCP focus groups concentrated on the process of the ARCP and the relevance of SLEs to this process. The data from the trainees and the trainers were kept separate during the analysis.

\section{Table 1. Timing of focus groups and questionnaires during the WPBA pilot study}

\section{Time}

September 2012

November 2012

December 2012

January 2013

April 2013

May 2013

July 2013

August 2013

\section{Focus groups}

Three trainee focus groups $(n=6,8,9)$

Three trainer focus groups $(n=4,8,2)$

WPBA $=$ workplace-based assessments

Three trainee focus groups $(n=3,5,8)$

Three trainer focus groups $(n=4,6,4)$

Three trainer focus groups $(n=4,4,2)$

One trainee focus group ( $n=4$ )

One trainer focus group $(n=5)$

\section{Questionnaires}

One trainee questionnaire $(n=165)$ One trainer questionnaire $(n=18)$

One trainee questionnaire $(n=50)$ One trainer questionnaire $(n=10)$

Three trainee focus groups $(n=4,25,4)$

One trainee questionnaire $(n=197)$

One trainer questionnaire $(n=35)$ 
The first questionnaire, which focused on the pre-pilot WPBAs, had responses from 165 trainees and 18 trainers. The second questionnaire, which focussed on the SLEs, had responses from 50 trainees and 10 trainers. The third questionnaire focused on the relevance of SLEs during the ARCP process. There were responses from 197 trainees and 35 trainers. The response rates of trainees in the three questionnaires were $19.3 \%, 5.8 \%$ and $23.0 \%$ (to 1 decimal point), respectively, whereas the trainers' response rates were $2.7 \%, 1.5 \%$ and $5.2 \%$ (to 1 decimal point), respectively. However, there were trainees and trainers who abstained from some of the questions in the questionnaires. Therefore, the total number of trainees and trainers who responded is stated under each relevant theme.

\section{Pre-pilot WPBAs}

The first questionnaire indicated that the pre-pilot WPBAs were rarely perceived as learning tools by the trainees. Only 37 out of 165 trainees felt that the pre-pilot WPBAs facilitated learning, whereas only eight out of 165 trainees felt that the pre-pilot WPBAs were helpful in identifying areas for further learning. This is supported by the result that only 44 out of 127 trainees received feedback regularly following a WPBA. Furthermore, only 17 out of the 127 trainees regularly had an action plan following a WPBA. The trainees predominately perceived the pre-pilot WPBAs as part of the requirements for progression at their ARCPs:

Trainee: '...general view amongst the trainees and consultants is that the current system of WPBA is a relatively pointless, 'tick-box' exercise...education and training is a complete afterthought in the current system...'

The trainers who responded in the first questionnaire perceived the pre-pilot WPBAs as assessments with an additional element of learning (Table 2). However, the trainers perceived the assessment component as the predominate component of the pre-pilot WPBAs (Table 2). Only six out of 18 trainers felt that the pre-pilot WPBAs facilitated learning, whereas none of the trainers felt that the pre-pilot WPBAs were helpful in identifying areas for improvement for their trainees. Furthermore, there were trainers who felt that the pre-pilot WPBAs were simply part of the requirements for the trainees' progression:

Trainer: '[A WPBA] Does nothing to demonstrate experience in practice and sadly, for many, is a tick box exercise.'

Trainer: '[Purpose of WPBAs] Evidence for ARCP.'
Nine out of 15 trainers felt that they would perform WPBAs with no planning. Therefore, there was no planning around these clinical encounters to maximise the learning.

\section{Implementation of workplace-based assessments}

The data from the focus groups and questionnaires demonstrated that WPBAs were often performed without direct observation regardless of the purpose and methods of the WPBAs. Therefore, those mini-CEXs that were undertaken without direct observation would become a non-observational assessment rather like a $\mathrm{CbD}$ :

Trainer: 'For about $80 \%$ of CEXs [mini-CEX], the consultant doesn't see the junior take the history.'

\section{Supervised learning events}

\section{Feasibility}

There were 23 trainees in the focus groups who responded on this topic. Eight out of the 23 trainees had difficulties in undertaking SLEs (Table 3). The findings for the trainers were similar to those for the trainees. There were 14 trainers who responded on this topic in the focus groups, five of whom had difficulties undertaking SLEs.

The results from the questionnaire were similar for both the trainees and the trainers. There were 41 trainees who responded on this topic and 11 of them had difficulties undertaking SLEs. There were only six responses from the trainers in the second questionnaire, three of whom had some difficulties in performing SLEs. This particular finding would need to be interpreted with a degree of caution in view of the small sample size.

The trainees' focus groups and questionnaires offered an insight into the difficulties encountered, which were mostly time or work pattern constraints or the disengagement of the trainers (Table 3). The trainers' focus groups and questionnaires also showed similar findings, in that the main difficulties were time or work pattern constraints or disengagement between the trainees and the trainers (Tables 3 and 4).

\section{Validity}

The focus groups demonstrated that the trainees and the trainers had a poor understanding of the purposes of SLEs (Table 3):

Trainee: 'Great confusion over SLEs...'

Trainer: 'I'll be honest, I get lost between the old system and the new system....and all the acronyms.'

Table 2. Frequency of comments and themes on pre-pilot WPBA in the first questionnaire

\begin{tabular}{|c|c|c|c|c|c|}
\hline Positive themes & Trainees & Trainers & Negative themes & Trainees & Trainers \\
\hline Enable feedback (educational value) & 27 & 3 & $\begin{array}{l}\text { Assessment with lesser learning element } \\
\text { (educational value) }\end{array}$ & 36 & 8 \\
\hline Facilitate learning (educational value) & 37 & 6 & $\begin{array}{l}\text { Act as requirements for progression (educational } \\
\text { value) }\end{array}$ & 85 & 9 \\
\hline $\begin{array}{l}\text { Identify areas for improvement } \\
\text { (educational value) }\end{array}$ & 8 & 0 & & & \\
\hline
\end{tabular}


Table 3. Frequency of comments and themes on SLEs from focus groups ${ }^{a}$

\begin{tabular}{|c|c|c|c|c|c|}
\hline Positive themes & Trainees & Trainers & Negative themes & Trainees & Trainers \\
\hline \multicolumn{6}{|l|}{ Themes on feasibility } \\
\hline \multirow[t]{3}{*}{ Ease in undertaking SLE (feasibility) } & \multirow[t]{3}{*}{15} & \multirow[t]{3}{*}{9} & Difficult with using SLE (feasibility) & 8 & 5 \\
\hline & & & Time constraint (feasibility) & 25 & 16 \\
\hline & & & Work pattern constraints (feasibility) & 13 & 1 \\
\hline \multicolumn{6}{|l|}{ Themes on SLEs as learning tools } \\
\hline $\begin{array}{l}\text { Learning tool (validity and educational } \\
\text { value) }\end{array}$ & 15 & 7 & $\begin{array}{l}\text { Assessment with lesser learning element } \\
\text { (validity and educational value) }\end{array}$ & 14 & 17 \\
\hline $\begin{array}{l}\text { Inform, and/or provide insight into, } \\
\text { progress (validity) }\end{array}$ & 29 & 19 & $\begin{array}{l}\text { Unable to inform, and/or provide insight } \\
\text { into, progress (validity) }\end{array}$ & 9 & 8 \\
\hline $\begin{array}{l}\text { Identify learning needs (validity and } \\
\text { educational value) }\end{array}$ & 19 & 6 & Poor understanding of SLE (validity) & 50 & 37 \\
\hline $\begin{array}{l}\text { Enable feedback (validity and educational } \\
\text { value) }\end{array}$ & 19 & 3 & $\begin{array}{l}\text { Inappropriate use of ACAT, CbD and/or } \\
\text { MiniCEX (validity) }\end{array}$ & 8 & 19 \\
\hline \multicolumn{6}{|l|}{ Themes on feedback and action plans in SLEs } \\
\hline \multirow{2}{*}{$\begin{array}{l}\text { Immediate feedback provided (educational } \\
\text { value) }\end{array}$} & \multirow[t]{2}{*}{34} & \multirow[t]{2}{*}{15} & No immediate feedback (educational value) & 14 & 0 \\
\hline & & & Non-specific feedback (educational value) & 14 & 0 \\
\hline $\begin{array}{l}\text { Formulation of action plan (educational } \\
\text { value) }\end{array}$ & 8 & 8 & No action plan made (educational value) & 15 & 10 \\
\hline \multicolumn{6}{|c|}{ Themes on the contribution of SLEs to the ARCP process } \\
\hline $\begin{array}{l}\text { Non-contribution to ARCP and/or } \\
\text { progression (validity) }\end{array}$ & 6 & 15 & $\begin{array}{l}\text { Should contribute to progression and/or } \\
\text { ARCP (validity) }\end{array}$ & 60 & 21 \\
\hline $\begin{array}{l}\text { Confidential between trainee and trainer } \\
\text { (validity) }\end{array}$ & 3 & 10 & & & \\
\hline
\end{tabular}

${ }^{a}$ Trainees = 76; trainers = 43. ACAT = acute care assessment tool; $A R C P=$ Annual Review of Competence Progression; $C b D=$ case-based discussion; MiniCEX = miniclinical evaluation exercise; SLE = supervised learning events.

Trainer: 'It took me four years...to get used to the previous system, and now it's all changing. People are just getting confused.'

The data from the second questionnaire resonated with the findings from the focus groups. The trainees reported that there was a poor understanding of the purpose of SLEs (Table 5):

Trainee: '....assessors are getting confused as to the purpose of it [SLEs]...'

Trainee: '...their [SLEs] role is unclear. Trainers or trainees don't seem to $[s i c]$ able to clearly define what constitutes SLEs...'

The trainers' questionnaire did not contain as many data on this topic because the questionnaire did not explicitly explore the subject.
The trainees in both the focus groups and the questionnaires perceived SLEs in their current format as learning tools (Tables 3 and 5):

Trainee: '[SLEs are] Formative, helpful to trainees...identify strengths and propose areas of improvement.'

Trainee: '[A SLE is a] Formative assessment...demonstrate your ability in different situations. Identify strengths and areas for improvement.'

This represents an important change in the perception of WPBAs. Furthermore, the data from the trainers in both the focus groups and questionnaires demonstrated that the trainers did view SLEs as learning tools. However, the change was less significant than it was with the trainees (Table 3 and 5):

Table 4. Frequency of comments on the engagement of trainees and trainers from results of focus groups and questionnaires

\section{Theme}

Variable engagement between trainees and trainers
Focus groups

Trainees $(\mathrm{n}=76)$

41
Second and third questionnaires

Trainees $(n=247)$

54

25 
Table 5. Frequency of comments on SLEs in the second and third questionnaires ${ }^{a}$

\begin{tabular}{|c|c|c|c|c|c|}
\hline Positive themes & Trainees & Trainers & Negative themes & Trainees & Trainers \\
\hline \multicolumn{6}{|l|}{ Themes on feasibility } \\
\hline & & & Time constraint (feasibility) & 2 & 1 \\
\hline & & & Work patterns constraint (feasibility) & 16 & 0 \\
\hline \multicolumn{6}{|l|}{ Themes on SLEs as learning tools } \\
\hline Learning tool (validity and educational impact) & 19 & 4 & $\begin{array}{l}\text { Assessment with lesser learning element } \\
\text { (validity and educational value) }\end{array}$ & 8 & 5 \\
\hline $\begin{array}{l}\text { Identify learning needs (validity and } \\
\text { educational value) }\end{array}$ & 22 & 0 & Poor understanding of SLE (validity) & 124 & 5 \\
\hline $\begin{array}{l}\text { Enable feedback (validity and educational } \\
\text { impact) }\end{array}$ & 36 & 4 & & & \\
\hline \multicolumn{6}{|c|}{ Themes on SLEs' contribution to the ARCP process } \\
\hline $\begin{array}{l}\text { Non-contribution to ARCP and/or progression } \\
\text { (validity) }\end{array}$ & 11 & 11 & $\begin{array}{l}\text { Should contribute to progression and/or } \\
\text { ARCP (validity) }\end{array}$ & 109 & 15 \\
\hline $\begin{array}{l}\text { Confidential between trainee and trainer } \\
\text { (validity) }\end{array}$ & 10 & 6 & & & \\
\hline
\end{tabular}

Trainer: '[SLEs are] to assess trainee's knowledge and performance...'

The trainees and trainers in the focus groups felt that SLEs provided them with an insight into the trainees' learning (Table 3):

Trainee: '[SLE] helps you understand your short comings and good things.'

Trainer: 'Good at picking up evidence of those who are very poor - useful...'

The second questionnaire demonstrated similar findings. Thirty-three out of 41 trainees felt that SLEs provided them with an insight into their learning. There were only six trainers who responded on this subject. Nonetheless, five of them felt that SLEs provided them with an insight into the trainees' learning.

\section{Educational value}

The trainees in the focus groups reported that the trainers would often provide immediate feedback following an SLE (Table 3). Most of this feedback was only provided verbally. The formal written feedback was usually provided at a later date (Table 3). Furthermore, the trainees felt that the feedback was often non-specific:

Trainee: 'Verbal [feedback] after the event immediately then written upon completion of the assessment. Depends. [Written feedback] Can be done next day or takes weeks to be completed.'

Trainee: 'Variable. Variable on QUALITY and CLARITY of issues that need improving.'

The trainers in the focus groups reported similar experiences to the trainees in terms of feedback (Table 3). Evidence from the focus groups demonstrated that both the trainees and trainers felt that the feedback provided was not regularly translated into an action plan (Table 3 ):
Trainer: 'I normally do [translate the outcomes of SLEs into action plan] - not always. Other trainers rarely.'

The data from the trainees' second questionnaire illustrated that six out of 41 trainees did not receive feedback following their SLEs. The questionnaire did not provide any further information on the feedback provided other than the fact that only 10 out of 35 trainees felt that the feedback that they had received was helpful. Furthermore, the questionnaire showed that only 15 out of the 41 trainees had an action plan formulated regularly following an SLE. The findings from the questionnaire are in keeping with those from the focus groups. Overall, the focus groups and questionnaires showed that feedback was either lacking, delayed or non-specific following SLEs. Furthermore, trainee and trainer disengagement was reported by both the trainees and the trainers as a common cause of ineffective feedback:

Trainee: 'My experience is...of consultants having little time or interest in making them a formative process.'

Trainer: 'Maybe allocate the clinical supervisor role to people who are enthusiasts.'

\section{Role of SLEs in ARCP decision-making}

The focus groups identified that a large proportion of the trainees felt that SLEs should contribute directly towards the ARCP process. The third questionnaire showed similar findings, where 127 out of 177 of trainees stated that they would like their SLEs to contribute directly towards the ARCP process. The trainees felt that a significant degree of effort had been invested in undertaking and learning through SLEs. Furthermore, the trainees felt that SLEs should demonstrate their progression and development during the training period:

Trainee: 'I think they [SLEs] should be viewed [by ARCP panel] - show progression over time...' 
Trainee: 'Definitely [for SLEs to contribute directly to ARCP]. In my case, they showed how I had progressed through the year and gave a wider picture of my experience and knowledge.'

Trainee: 'I think the journey of completing a competency is as important as completing it.'

In the third questionnaire, there were several trainees who felt that a review of their SLEs by the ARCP panel would not influence the quality of comments from their trainers:

Trainee: 'Keeping the comments [of SLEs] inaccessible to the ARCP panel hasn't changed the quality of the feedback in my experience.'

The focus groups illustrated similar findings to the questionnaire. The trainees felt that SLEs should contribute directly to the ARCP:

Trainee: 'Yes definitely [for SLEs to contribute directly to ARCP]. Good assessment of progress through the year.'

The trainers in the focus groups felt similarly to the trainers in the questionnaire. The third questionnaire identified 20 trainers who felt that SLEs should contribute directly towards the ARCP process. However, there were trainers who felt that SLEs should not contribute directly towards the ARCP process. They felt that this would make the trainers more apprehensive in providing feedback. There was a third group of trainers who felt that SLEs should remain confidential between the trainees and their supervisors. These trainers suggested that SLEs should contribute towards the ARCP process for trainees who are underperforming. The intended benefit is to preserve the confidentiality of SLEs between trainees and trainers to an extent and, thus, the nature of the feedback. The trainers who suggested this felt that this would not impact on the trainers' ability to provide constructive feedback:

Trainer: 'It [SLE contributing to ARCP] is useful if candidates have difficulties; to see improvement. Useful for [the] panel to see candidate has had input.'

\section{Discussion}

\section{Pre-pilot WPBAs}

Most of the trainees and trainers rarely perceived the pre-pilot WPBAs as learning tools. Furthermore, there were only a few trainees who regularly received specific feedback and action plans following their WPBAs. WPBAs were often undertaken without deliberate planning, which can lead to less effective feedback. ${ }^{13,14}$

SLEs were seen as having the potential to address issues associated with the pre-pilot WPBAs. However, the study showed that several of these issues persisted despite the changes. This is discussed in more detail below.

\section{Implementation of WPBAs within the pilot}

The focus groups and the second questionnaire demonstrated that WPBAs were often performed without direct observation. Any mini-CEXs that were performed without direct observation would become a non-observational assessment, rather like CbDs.
The above is an important finding because mini-CEXs are intended to promote direct observation of trainees and this has a vital part in enhancing learning in the clinical environment. ${ }^{7,9,15,16}$ Nonetheless, this suggests that there was either poor understanding of the methods of WPBAs or the trainees and the trainers did not adhere to the methods. Regardless of the underlying cause, the inappropriate use of SLEs would have diminished the validity and educational value of SLEs.

\section{SLES}

\section{Feasibility}

The focus groups and second questionnaire showed that the trainees and trainers had some difficulties in undertaking SLEs. The trainees and the trainers felt that time constraints, work patterns and trainee-trainer disengagement were the most common barriers in undertaking SLEs.

\section{Validity}

The focus groups and questionnaires both showed that the trainees' perception of SLEs was different from that of the prepilot WPBAs. The trainees and trainers perceived SLEs to be intended as learning tools. The trainees and the trainers in both the focus groups and the second questionnaire perceived SLEs as having the potential to provide them with an insight into a trainee's learning and development.

\section{Educational value}

The focus groups and questionnaires identified that, despite the perception that SLEs are learning tools, there was either a lack of feedback or it was delayed or non-specific following SLEs. Feedback has an important role in enabling WPBAs to facilitate learning. ${ }^{11,15,17-19}$ Therefore, feedback should be relevant and specific to encourage self-evaluation and self-reflection. ${ }^{11,14,15,20}$ Furthermore, trainees are less likely to value non-specific feedback. ${ }^{13}$ Trainees should routinely formulate action plans and learning goals in discussion with their trainers following SLEs because these facilitate trainees learning. ${ }^{11,13-15}$ However, the focus groups and questionnaires showed that action plans were not regularly formulated following SLEs. These findings are important because trainees consider feedback to be one of the most important components of WPBAs. ${ }^{19}$

Pelgrim et $\mathrm{al}^{14}$ found that the inclusion of a feedback tool alone did not improve feedback. Appropriate guidance and training for both the trainers and trainees are required to improve the feedback process. ${ }^{14,21,22}$ Trainee-trainer disengagement might also have affected the effectiveness of feedback. ${ }^{13,21,22}$ Disengagement would have impeded trainees and trainers in identifying and agreeing on trainee-directed learning goals and planning of WPBAs. The latter is important because Pelgrim et al $^{14}$ identified that effective feedback requires deliberate planning for WPBAs. This is supported by the evidence that the pilot WPBAs were often undertaken with no prior arrangement between the trainees and the trainers. Furthermore, disengagement would have prevented trainers from encouraging trainees to actively seek feedback.

Despite the changes in the trainees' and trainers' perception of SLEs, the evidence suggests that SLEs did not yield effective feedback and action plans. This, in turn, suggests that 
SLEs were not as effective in promoting self-reflection and development of the trainees as they could be. ${ }^{11,13,14}$ Overall, the evidence suggests that SLEs were not effective as learning tools and were unable to address all the issues of the pre-pilot WPBAs as identified in the first questionnaire.

Role of SLEs in ARCP decision making

The trainees felt that a significant degree of effort had been invested in undertaking SLEs. Furthermore, the trainees felt that SLEs should demonstrate their development and progression. Therefore, trainees in the focus groups and the questionnaires felt that SLEs should contribute directly towards the ARCP decision process.

The focus group and third questionnaire showed that the trainers were slightly more divided over this issue. There were trainers who were concerned that the feedback process would become ineffective if SLEs contributed directly towards the ARCP process. Therefore, there were trainers who suggested that only the SLEs of trainees who were having difficulties with their development should contribute directly towards their ARCPs. However, there were trainees who felt that a review of their SLEs by the ARCP panel would have not influenced the feedback provided.

The key finding from the discussion above is that the trainees and trainers would like several assessment tools to be utilised to demonstrate the trainees' development. This resonates with the concept that several assessment tools undertaken over a period of time with a variety of scenarios should be utilised to demonstrate longitudinal progression because a single summative assessment would not be sufficiently reliable to provide judgement on a trainee's performance..$^{10,17,23-26}$ It is important to make the purpose of SLEs in demonstrating longitudinal progression clear to the trainees.

\section{Lessons learnt from pilot}

\section{WPBAs as learning tools}

The trainees and the trainers rarely perceived or utilised the pre-pilot WPBAs as learning tools. Therefore, the pre-pilot WPBAs were less effective as assessments for learning.

Lesson learnt: it is important to encourage trainees and trainers to view WPBAs or any assessment for learning as learning tools.

\section{Methods of WPBAs}

Despite the guidance provided during the pilot, WPBAs were often performed without direct observation regardless of their intended purposes and method. Direct observation has an important role in facilitating learning in the clinical environment. ${ }^{16}$ Furthermore, any mini-CEX that was performed without direct observation would have diminished validity and educational value and became effectively a CbD.

Lesson learnt: it is important to provide clear guidance and training on the methods and purposes of WPBAs. However, this alone might be insufficient. Further work to explore and address the cause(s) for the lack of direct observation would be of benefit.

\section{Educational value of SLEs}

The pilot was successful in promoting the trainees' and the trainers' views on SLEs as learning tools. However, despite the perception of SLEs as learning tools, there was often no or ineffective feedback. Furthermore, action plans were not formulated regularly following SLEs.

There were trainees and trainers who felt that SLEs should contribute towards the ARCP process. They felt that SLEs should demonstrate the trainees' development, but did not feel that this would impede feedback or diminish the potential education value of SLEs.

Lessons learnt: it is of benefit to demonstrate and reinforce the role of feedback and action plans in enhancing learning in WPBAs and assessments for learning. This should be taking into consideration in any plans to promote and reinforce the concept of assessments for learning as learning tools. In addition, it is important to provide clear guidance and training on providing effective feedback to enable assessments for learning to be effective. Trainees and trainers feel that assessments for learning are able to demonstrate longitudinal progression and development of trainees. Consideration should be given to the role that any assessment for learning should have in the overall assessment of trainees. However, this must be done with caution to avoid such assessments becoming assessments of learning.

\section{Trainee and trainer engagement}

Trainee and trainer disengagement had an important role in impeding the use and impact of SLEs as part of the learning process. It was shown to be one of the major difficulties in undertaking SLEs in the clinical environment in the focus groups and the questionnaires. Furthermore, the focus groups and the questionnaires demonstrated that there was a lack of, or nonspecific, feedback and action plans following SLEs. Disengagement would have probably had a role in this (see above). The trainees and trainers were both aware of the impact of disengagement although disengagement persisted during the pilot.

Lessons learnt: trainee and trainer engagement has an important role in improving the positive impact of any assessment for learning. However, trainee and trainer disengagement contributes towards ineffective feedback in assessments for learning. Therefore, it is important to encourage trainee and trainer engagement. However, trainees and trainers are often aware of the consequences of disengagement. Thus, any intervention that simply promotes the benefits of engagement would be unlikely to succeed. Successful interventions, namely workshops for supervisors with accreditation, would also need to promote a cultural change.

\section{Limitations}

The overall sample sizes for the focus groups and questionnaires were variable. There were small sample sizes for the ARCP focus groups and the trainers' second questionnaire. However, the study did not collect any data on the demographics and background of the participants in the focus groups and the questionnaires. Therefore, the study is unable 
to provide any evidence to ascertain whether the sampled population was a good representation of all the participants in the pilot. This should be taken into consideration when interpreting the findings and further research in this area would be of benefit. Nonetheless, all the participants in all three regions in the pilot were invited to take part in the focus groups and the questionnaires. There was also a high degree of triangulation between the focus groups and the questionnaires. Thus, it would appear that those smaller sample sizes did not have a significant impact on the findings of the pilot.

There was possibly an element of selection bias with those participating in the focus groups and the questionnaires. The trainees and trainers who participated were more likely to be either enthusiasts or the opposite. This must be taken into consideration when interpreting and applying the findings of this study.

\section{Conclusion}

The study has provided insight into the implementation and impact of SLEs. The lessons learnt through the pilot are likely to be relevant to other assessments for learning. Therefore, these lessons learnt, which include trainee and trainer engagement, effective feedback and the overall role of assessments for learning in the assessment of trainees, should be taken into consideration when implementing any assessment for learning. Further work into promoting and reinforcing direct observation is recommended to provide interesting insights into assessment for learning in the clinical environment.

\section{References}

1 General Medical Council. Learning and assessment in the clinical environment: the way forward. London: GMC, 2011.

2 Wood D. Formative assessment. Edinburgh: ASME, 2007.

3 Black P, Harrison C, Lee C, Marshall B, Wiliam D. Working inside the black box: assessment for learning in the classroom. Phi Delta Kappan 2004;86:8-21.

4 Royal College of Physicians. Workplace-based assessment pilot. London: RCP, 2012.

5 Norcini J, Blank L, Arnold G and Kimball HR. The mini-CEX (clinical evaluation exercise): A preliminary investigation. Ann Inter Med 1995;123:795-9.

6 Norcini J, Blank L, Duffy F, Fortna GS. The mini-CEX: a method for assessing clinical skills. Ann Inter Med 2003;138:476-81.

7 Holmboe E, Huot S, Chung J, Norcini J, Hawkins RE. Construct validity of the miniclinical evaluation exercise (MiniCEX). Acad Med 2003;78:826-30.

8 Johnson G, Barrett J, Jones M, Parry D, Wade W. Feedback from educational supervisors and trainees on the implementation of curricula and the assessment system for core medical training. Clin Med 2008;8:484-9.
9 Kogan J, Holmboe E, Hauer K. Tools for direct observation and assessment of clinical skills of medical trainees. JAMA 2009;302:1316-26.

10 Wilkinson J, Crossely J, Wragg A et al. Implementing workplacebased assessment across the medical specialties in the United Kingdom. Med Educ 2008;42:364-73.

11 Norcini J, Burch V. Workplace-based assessment as an educational tool: AMEE Guide No. 31. Med Teach 2007;29:855-71.

12 Cohen L, Manion L, Morrison K. Research methods in education. Abingdon: Routledge, 2011.

13 Sargeant J, Eva KW, Armson H et al. Features of assessment learners use to make informed self-assessments of clinical performance. Med Educ 2011;45:636-47.

14 Pelgrim EA, Kramer AW, Mokkink H, van der Vleuten CP. The process of feedback in workplace-based assessment: organisation, delivery, continuity. Med Educ 2012;46:604-12.

15 van de Ridder JM, Stokking KM, McGaghie WC, ten Cate OT. What is feedback in clinical education? Med Educ 2008;42:189-97.

16 Norcini J. The power of feedback. Med Educ 2010;44:16-7.

17 Swanwick T, Chana N. Workplace-based assessment. Br J Hosp Med 2009;70:290-3.

18 Miller A, Archer J. Impact of workplace based assessment on doctors' education and performance: a systemic review. BMJ 2010;341:c5064.

19 Seadon H, Salleh S, Balakrishnan A, Imray CHE, Saedon M. The role of feedback in improving the effectiveness of workplace based assessments: a systematic review. BMC Med Educ 2012;12:25.

20 Norcini J, Anderson B, Bollela V et al. Criteria for good assessment: consensus statement and recommendations from the Ottawa 2010 Conference. Med Teach 2011;33:206-14.

21 Bindal T, Wall D, Goodyear H. Trainee doctors' views on workplacebased assessments: Are they just a tick box exercise? Med Teach 2011;33:919-27.

22 Mehta F, Brown J, Shaw NJ. Do trainees value feedback in casebased discussion assessments? Med Teach 2013;35:e1166-72.

23 Crossley J, Johnson G, Booth J, Wade W. Good questions, good answers: construct alignment improves the performances of workplace-based assessment scales. Med Educ 2011;45:560-9.

24 Driessen E, Scheele F. What is wrong with assessment in postgraduate training? Lessons from clinical practice and educational research. Med Teach 2013;35:569-74.

25 Mamelok J. Developing assessments for enhanced specialty training programmes for licensing in general practice. Educ Primary Care 2013;24:230-3.

26 Rughani A. Workplace-based assessment and the art of performance. Br J Gen Pract 2008;58:1377-80.

27 Al-Kardi HM, Al-Kadi MT, van der Vleuten CPM. Workplace-based assessment and students' approaches to learning: a qualitative inquiry. Med Teach 2013;35:S31-8.

Address for correspondence: Mrs W Wade, Education Department, Royal College of Physicians, 11 St Andrews Place, London, NW1 4LE, UK.

Email:winnie.wade@rcplondon.ac.uk 